\title{
Upper and Lower Bounds for Essential Norm of Weighted Composition Operators from Bergman Spaces with Békollé Weights
}

\author{
Elina Subhadarsini ${ }^{1}$ and Ajay K. Sharma $\mathbb{B D}^{2}$ \\ ${ }^{1}$ Department of Mathematics, Shri Mata Vaishno Devi University, Kakryal, Katra 182320, India \\ ${ }^{2}$ Department of Mathematics, Central University of Jammu, Rahya-Suchani (Bagla) Samba, 181143 Jammu, J \& K, India
}

Correspondence should be addressed to Ajay K. Sharma; aksju_76@yahoo.com

Received 10 June 2020; Revised 5 November 2020; Accepted 3 December 2020; Published 29 December 2020

Academic Editor: John R. Akeroyd

Copyright (c) 2020 Elina Subhadarsini and Ajay K. Sharma. This is an open access article distributed under the Creative Commons Attribution License, which permits unrestricted use, distribution, and reproduction in any medium, provided the original work is properly cited.

Let $\sigma$ be a weight function such that $\sigma /\left(1-|z|^{2}\right)^{\alpha}$ is in the class $B_{p_{0}}(\alpha)$ of Békollé weights, $\mu$ a normal weight function, $\psi$ a holomorphic map on $\mathbb{D}$, and $\varphi$ a holomorphic self-map on $\mathbb{D}$. In this paper, we give upper and lower bounds for essential norm of weighted composition operator $W_{\psi, \varphi}$ acting from weighted Bergman spaces $\mathscr{A}^{p}(\sigma)$ to Bloch-type spaces $\mathscr{B}_{\mu}$.

\section{Introduction and Preliminaries}

Let $\mathbb{D}$ be the open unit disk in the complex plane $\mathbb{C}$ and $H(\mathbb{D})$ the space of all holomorphic functions on $\mathbb{D}$. For a $\psi \in H(\mathbb{D})$ and $\varphi$ a holomorphic self-map of $\mathbb{D}$, the weighted composition operator $W_{\psi, \varphi}$ is a linear operator on $H(\mathbb{D})$ defined by $W_{\psi, \varphi} f=\psi(f \circ \varphi), f \in H(\mathbb{D})$. Several authors have studied these weighted composition operators on different spaces of analytic functions, see for example, [1-12] and the related references therein. Recently, Stevic and Sharma [12] characterized boundness and compactness of $W_{\psi, \varphi}$ acting from weighted Bergman spaces $\mathscr{A}^{p}(\sigma)$ to Bloch-type spaces $\mathscr{B}_{\mu}$. Motivated by results in [12], in this paper, we give upper and lower bounds for essential norm of a weighted composition operator acting between these spaces.

A continuous function $\sigma:[0,1) \longrightarrow[0, \infty)$ is called a weight or a weight function. We extend it on $\mathbb{D}$ by defining $\sigma(z)=\sigma(|z|)$ for all $z \in \mathbb{D}$. For $0<p<\infty$ and $\sigma$ a weight, denoted by $\mathscr{A}^{P}(\sigma)$ the weighted Bergman space consisting of holomorphic functions $f$ on $\mathbb{D}$ such that

$$
\|f\|_{\mathscr{A}^{p}(\sigma)}^{p}=\int_{\mathbb{D}}|f(z)|^{p} \sigma(z) d A(z)<\infty,
$$

where $d A$ is the normalized area measure in $\mathbb{D}$. If $\sigma(z)=\sigma_{\gamma}$ $(z)=\left(1-|z|^{2}\right)^{\gamma}(\gamma>-1)$, then $\mathscr{A}^{p}(\sigma)$ is the well-known weighted Bergman space $A_{\gamma}^{p}$.

For $p_{0}>1$ and $\alpha>-1$, the class $B_{p_{0}}(\alpha)$ of Békollé weights consists of weights $\sigma$ with the property that there exists a constant $C>0$ such that

$$
\left(\int_{S(\theta, h)} \sigma d A_{\alpha}\right) \leq C\left[A_{\alpha}(S(\theta, h))\right]^{p_{0}}\left(\int_{S(\theta, h)} \sigma^{p^{\prime} 0} 1 p_{0} d A_{\alpha}\right)^{-p_{0} / p_{0}^{\prime}} .
$$

Here, $\alpha>-1, d A_{\alpha}(z)=(\alpha+1)\left(1-|z|^{2}\right)^{\alpha} d A(z)$ is the probability measure on $\mathbb{D}, S(\theta, h)=\left\{z=r e^{i \phi}: 1-h<r<1,|\theta-\phi|\right.$ $<h / 2\}, \theta \in[0,2 \pi], h \in(0,1)\}$ is the Carleson square in $\mathbb{D}$, and $p^{\prime}{ }_{0}$ is the conjugate exponent of $p_{0}$, that is, $1 / p_{0}+1 / p^{\prime}{ }_{0}=1$. Recall that a weight $\mu$ is normal if there exist positive numbers $\eta$ and $\tau, 0<\eta<\tau$, and $\delta \in[0,1)$ such that

$$
\begin{aligned}
& \frac{\mu(r)}{(1-r)^{\eta}} \text { is decreasing on }[\delta, 1) \text { and } \lim _{r \rightarrow 1} \frac{\mu(r)}{(1-r)^{\eta}}=0, \\
& \frac{\mu(r)}{(1-r)^{\tau}} \text { is increasing on }[\delta, 1) \text { and } \lim _{r \rightarrow 1} \frac{\mu(r)}{(1-r)^{\tau}}=\infty .
\end{aligned}
$$


It is well known that classical weights $\sigma_{\alpha}(z)=\left(1-|z|^{2}\right)^{\alpha}$, $\alpha>-1$ are normal weights.

For a normal weight $\mu$, the weighted Bloch-type space $\mathscr{B}_{\mu}$ on $\mathbb{D}$ is the space of all functions $f$ in $H(\mathbb{D})$ such that $\sup _{z \in \mathbb{D}} \mu(z)\left|f^{\prime}(z)\right|<\infty$. The space $\mathscr{B}_{\mu}$ is a Banach space with the norm

$$
\|f\|_{\mathscr{B}_{\mu}}=|f(0)|+\sup _{z \in \mathbb{D}} \mu(z)\left|f^{\prime}(z)\right| .
$$

Throughout this paper, $r \in(0,1)$ is fixed, $p>0, p_{0}>1$, and $\eta>-1$. We also assume that $p_{0} \geq p, \sigma$ a weight function such that $\sigma /\left(1-|z|^{2}\right)^{\alpha}$ belongs to $B_{p_{0}}(\alpha), \gamma \geq(\eta+2) p_{0} / p-2$, and $K_{\lambda}^{\gamma}=1 /(1-\bar{\lambda} z)^{\gamma+2}$ be the reproducing kernel of the Bergman space $A^{p}\left(\sigma_{\gamma}\right)$. Constants are denoted by $C$; they are positive and not necessarily the same at each occurrence. The notation $A \lesssim B$ means that $A$ is less than or equal to a constant multiple of $B$, and $D \gtrsim E$ means that a constant multiple of $D$ is greater than or equal to $E$. When $A \lesssim B$ as well as $A \gtrsim B$, then we write $A \approx B$.

\section{Essential Norm of $W_{\psi, \varphi}: \mathscr{A}^{p}(\sigma) \longrightarrow \mathscr{B}_{\mu}$}

In this section, we give upper and lower bounds for the essential norm of weighted composition operator $W_{\psi, \varphi}: \mathscr{A}^{P}(\sigma)$ $\longrightarrow \mathscr{B}_{\mu}$.

Recall that if $X$ and $Y$ are two Banach spaces, then the essential norm $\|T\|_{e, X \rightarrow Y}$ of a bounded linear operator $T: X$ $\longrightarrow Y$ is defined as

$$
\|T\|_{e, X \rightarrow Y}=\inf \{\|T-K\|: K \text { is compact from } X \text { to } Y\},
$$

where $\|T\|$ denotes the usual operator norm. Clearly, $T$ is compact if and only if $\|T\|_{e, X \rightarrow Y}=0$.

Theorem 1. Let $\in(1, \infty), \psi \in H(\mathbb{D})$, and $\varphi$ be a holomorphic self-map of $\mathbb{D}$ such that $\|\varphi\|_{\infty}=1$. Assume that $W_{\psi, \varphi}: \mathscr{A}^{P}(\sigma)$ $\longrightarrow \mathscr{B}_{\mu}$ is bounded. Then,

$$
\begin{aligned}
\left\|W_{\psi, \varphi}\right\|_{e, \mathscr{A}^{p}(\sigma) \rightarrow \mathscr{B}_{\mu}}= & \limsup _{|\varphi(z)| \rightarrow 1} \mu(z)\left|\psi^{\prime}(z)\right|\left(\int_{D_{\varphi(z)}(r)} \sigma d A\right)^{-1 / p} \\
& +\limsup _{|\varphi(z)| \rightarrow 1} \frac{\mu(z)\left|\psi(z) \varphi^{\prime}(z)\right|}{1-|\varphi(z)|^{2}}\left(\int_{D_{\varphi(z)}(r)} \sigma d A\right)^{-1 / p},
\end{aligned}
$$

where $D_{\varphi(z)}(r)=\{w \in \mathbb{D}:|w-\varphi(z)|<r(1-|\varphi(z)|)\}$.

To prove the main result of this paper, we need the following lemmas. The next two lemmas can be found in [12].

Lemma 2. The following estimates hold:

(1) For each $z \in \mathbb{D}$, we have that

$$
\left|f^{(k)}(z)\right| \leq C \frac{\left(\int_{D_{z}(r)} \sigma d A\right)^{-1 / p}}{\left(1-|z|^{2}\right)^{k}}\|f\|_{\mathscr{A}^{p}(\sigma)} \text { for all } f \in A_{\sigma}^{p}
$$

(2) For each $\lambda \in \mathbb{D}$, we have that

$$
\left\|K_{\lambda}^{\gamma}\right\|_{A^{p}(\sigma)}=\frac{\left(\int_{D_{\lambda}(r)} \sigma d A\right)^{1 / p}}{\left(1-|\lambda|^{2}\right)^{\gamma+2}}
$$

where $D_{\lambda}(r)=\{z \in \mathbb{D}:|z-\lambda|<r(1-|\lambda|)\}$

Lemma 3. For each $\lambda \in \mathbb{D}$, the function $f_{\lambda}$ defined as

$$
f_{\lambda}(z)=\frac{\left(1-|\lambda|^{2}\right)^{1+(\eta+2) p_{0} / p}}{(1-\bar{\lambda} z)^{1+(\eta+2) p_{0} / p}}\left(\int_{D_{\lambda}(r)} \sigma d A\right)^{-1 / p}
$$

is in $A^{p}(\sigma)$. Moreover, $\sup _{\lambda \in \mathbb{D}}\left\|f_{\lambda}\right\|_{A^{p}(\sigma)}=1$ and $f_{\lambda}$ converges to zero, uniformly on compact subsets of $\mathbb{D}$ as $|\lambda| \longrightarrow 1$.

The next lemma can be found in [8].

Lemma 4. Let $p \in(1, \infty)$. If a bounded sequence $\left\{f_{k}\right\}_{k \in \mathbb{N}}$ in $\mathscr{A}^{P}(\sigma)$ converges to 0 uniformly on compact subsets of $\mathbb{D}$, then $\left\{f_{k}\right\}_{k \in \mathbb{N}}$ also converges to 0 weakly in $\mathscr{A}^{p}(\sigma)$.

Proof of Theorem 1. Lower bound. Let $\left\{\zeta_{j}\right\}_{j \in \mathbb{N}}$ be a sequence in $\mathbb{D}$ such that $\left|\varphi\left(\zeta_{j}\right)\right| \longrightarrow 1$ as $j \longrightarrow \infty$ and

$$
\begin{array}{r}
\limsup _{j \rightarrow \infty} \mu\left(\zeta_{j}\right)\left|\psi^{\prime}\left(\zeta_{j}\right)\right|\left(\int_{D_{\varphi\left(\zeta_{j}\right)}(r)} \sigma d A\right)^{-1 / p} \\
\quad=\limsup _{|\varphi(z)| \rightarrow 1} \mu(z)\left|\psi^{\prime}(z)\right|\left(\int_{D_{\varphi(z)}(r)} \sigma d A\right)^{-1 / p} .
\end{array}
$$

For each $j \in \mathbb{N}$, let $\gamma_{j}$ be defined as $\gamma_{j}(z)=1-(1-$ $\left.\left|\varphi\left(\zeta_{j}\right)\right|^{2}\right)\left(1-\varphi\left(\bar{\zeta}_{j}\right) z\right)^{-1}$. Then, $\gamma_{j} \in H^{\infty}$ and $\sup _{j \in \mathbb{N}}\left|\gamma_{j}(z)\right| \leq$ 3. Consider the family of functions defined as $g_{j}(z)=\gamma_{j}(z)$ $f_{\varphi\left(\zeta_{j}\right)}(z)$, where $f_{\varphi\left(\zeta_{j}\right)}$ is defined as in (9). Also, by Lemma 3, $\sup _{j}\left\|g_{j}\right\|_{A^{p}(\sigma)} \lesssim 1$ and $\left\{g_{j}\right\}_{n \in \mathbb{N}}$ converges to zero uniformly on compact subsets of $\mathbb{D}$ as $j \longrightarrow \infty$. By Lemma $4, g_{j}$ converges to zero weakly in $A^{P}(\sigma)$. Thus, for any compact operator $K: A^{p}(\sigma) \longrightarrow \mathscr{B}_{\mu}$, we have that $\left\|K g_{j}\right\|_{\mathscr{B}_{\mu}} \longrightarrow 0$ as $j \longrightarrow \infty$. Moreover, 


$$
\begin{aligned}
& \gamma_{j}\left(\varphi\left(\zeta_{j}\right)\right)=0, \gamma_{j}^{\prime}(z)=-\varphi\left(\bar{\zeta}_{j}\right) \frac{1-\left|\varphi\left(\zeta_{j}\right)\right|^{2}}{\left(1-\varphi\left(\bar{\zeta}_{j}\right) z\right)^{2}}, \\
& \gamma_{j}^{\prime}\left(\varphi\left(\zeta_{j}\right)\right)=\frac{-\varphi\left(\bar{\zeta}_{j}\right)}{1-\left|\varphi\left(\zeta_{j}\right)\right|^{2}} .
\end{aligned}
$$

Also, $g_{j}\left(\varphi\left(\zeta_{j}\right)\right)=0$ and

$$
f_{\varphi\left(\zeta_{j}\right)}\left(\varphi\left(\zeta_{j}\right)\right)=\left(\int_{D_{\varphi\left(\zeta_{j}\right)}(r)} \sigma d A\right)^{-1 / p}
$$

Now

$$
\begin{aligned}
f_{\varphi\left(\zeta_{j}\right)}(z)= & \varphi\left(\bar{\zeta}_{j}\right)\left((\eta+2) \frac{p_{0}}{p}+1\right)\left(\int_{D_{\varphi\left(\zeta_{j}\right)}(r)} \sigma d A\right)^{-1 / p} \\
& \cdot \frac{\left(1-\left|\varphi\left(\zeta_{j}\right)\right|^{2}\right)^{1+(\eta+2) p_{0} / p}}{\left(1-\varphi\left(\bar{\zeta}_{j}\right) z\right)^{2+(\eta+2) p_{0} / p}} f_{\varphi\left(\zeta_{j}\right)}\left(\varphi\left(\zeta_{j}\right)\right) \\
= & \varphi\left(\bar{\zeta}_{j}\right)\left((\eta+2) \frac{p_{0}}{p}+1\right) \frac{\left(\int_{D_{\varphi\left(\zeta_{j}\right)}(r)} \sigma d A\right)^{-1 / p}}{1-\left|\varphi\left(\zeta_{j}\right)\right|^{2}} .
\end{aligned}
$$

Therefore, from (12) and (13), we have that

$$
\begin{aligned}
g_{j}^{\prime}\left(\varphi\left(\zeta_{j}\right)\right) & =\gamma_{j}^{\prime}\left(\varphi\left(\zeta_{j}\right)\right) f_{\varphi\left(\zeta_{j}\right)}\left(\varphi\left(\zeta_{j}\right)\right)+\gamma_{j}\left(\varphi\left(\zeta_{j}\right)\right) f_{\varphi\left(\zeta_{j}\right)}\left(\varphi\left(\zeta_{j}\right)\right) \\
& =-\varphi\left(\bar{\zeta}_{j}\right) \frac{\left(\int_{D_{\varphi\left(\zeta_{j}\right)}(r)} \sigma d A\right)^{-1 / p}}{1-\left|\varphi\left(\zeta_{j}\right)\right|^{2}} .
\end{aligned}
$$

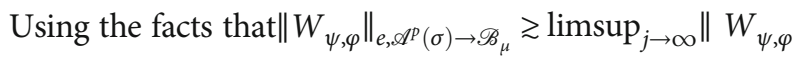
$g_{j}-K g_{j} \|_{\mathscr{B}_{\mu}}$ and

$\limsup _{j \rightarrow \infty}\left\|K g_{j}\right\|_{\mathscr{B}_{\mu}}=0$, we have that

$\left\|W_{\psi, \varphi}\right\|_{e, \mathscr{A}^{p}(\sigma) \rightarrow \mathscr{B}_{\mu}} \geq \limsup _{j \rightarrow \infty} \mu\left(\zeta_{j}\right)\left|\psi^{\prime}(\zeta) g_{j}\left(\varphi\left(\zeta_{j}\right)\right)+\psi\left(\zeta_{j}\right) \varphi^{\prime}\left(\zeta_{j}\right) g_{j}^{\prime}\left(\varphi\left(\zeta_{j}\right)\right)\right|$

$$
\geq \limsup _{j \rightarrow \infty} \mu\left(\zeta_{j}\right)\left|\psi\left(\zeta_{j}\right) \varphi^{\prime}\left(\zeta_{j}\right)\right| \frac{\left(\int_{D_{\varphi\left(\zeta_{j}\right)(r)}} \sigma d A\right)^{-1 / p}}{1-\left|\varphi\left(\zeta_{j}\right)\right|^{2}} .
$$

Again, let $\zeta_{j}$ be a sequence in $\mathbb{D}$ such that $\left|\varphi\left(\zeta_{j}\right)\right| \longrightarrow 1$ as $j \longrightarrow \infty$ and

$$
\begin{aligned}
& \lim _{j \rightarrow \infty} \mu\left(\zeta_{j}\right)\left|\psi\left(\zeta_{j}\right) \varphi^{\prime}\left(\zeta_{j}\right) \varphi\left(\zeta_{j}\right)\right|\left(\int_{D_{\varphi\left(\zeta_{j}\right)}(r)} \sigma d A\right)^{-1 / p} \\
& \quad=\limsup _{|\varphi(z)| \rightarrow 1} \mu(z)\left|\psi(z) \varphi^{\prime}(z) \varphi(z)\right|\left(\int_{D_{\varphi(z)}(r)} \sigma d A\right)^{-1 / p} .
\end{aligned}
$$

For each $j \in \mathbb{N}$, let $h_{j}$ be defined as

$$
\begin{aligned}
h_{j}(z)= & \left\{\frac{1}{1+(\eta+2) p_{0} / p} \frac{\left(1-\left|\varphi\left(\zeta_{j}\right)\right|^{2}\right)^{1+(\eta+2) p_{0} / p}}{\left(1-\varphi\left(\bar{\zeta}_{j}\right) z\right)^{1+(\eta+2) p_{0} / p}}\right. \\
& \left.-\frac{1}{2+(\eta+2) p_{0} / p} \frac{\left(1-\left|\varphi\left(\zeta_{j}\right)\right|^{2}\right)^{2+(\eta+2) p_{0} / p}}{\left(1-\varphi\left(\bar{\zeta}_{j}\right) z\right)^{2+(\eta+2) p_{0} / p}}\right\} \\
& \cdot\left(\int_{D_{\varphi\left(\zeta_{j}\right)}(r)} \sigma d A\right)^{-1 / p} .
\end{aligned}
$$

Then, by Lemma $3, \sup _{j}\left\|h_{j}\right\|_{A^{p}(\sigma)} \leqslant 1$ and $\left\{h_{j}\right\}_{n \in \mathbb{N}}$ converges to zero uniformly on compact subsets of $\mathbb{D}$ as $j \longrightarrow$ $\infty$. By Lemma $4, h_{j}$ converges to zero weakly in $A^{p}(\sigma)$. Thus, for any compact operator $K: A^{p}(\sigma) \longrightarrow \mathscr{B}_{\mu}$, we have that $\|$ $K h_{j} \|_{\mathscr{B}_{\mu}} \longrightarrow 0$ as $j \longrightarrow \infty$. Moreover, $h_{j}^{\prime}\left(\varphi\left(\zeta_{j}\right)\right)=0$ and

$$
h_{j}\left(\varphi\left(\zeta_{j}\right)\right)=\frac{1}{\left(1+(\eta+2) p_{0} / p\right)\left(2+(\eta+2) p_{0} / p\right)}\left(\int_{D_{\varphi\left(\zeta_{j}\right)}(r)} \sigma d A\right)^{-1 / p}
$$

Thus, using (18), we have that

$$
\begin{aligned}
& \left\|W_{\psi, \varphi}\right\|_{e, \not \mathscr{A}^{p}(\sigma) \rightarrow \mathscr{S}_{\mu}} \geq \limsup _{j \rightarrow \infty} \mu\left(\zeta_{j}\right) \mid \psi^{\prime}\left(\zeta_{j}\right) h_{j}\left(\varphi\left(\zeta_{j}\right)\right) \\
& +\psi\left(\zeta_{j}\right) \varphi^{\prime}\left(\zeta_{j}\right) h_{j}^{\prime}\left(\varphi\left(\zeta_{j}\right)\right) \text { । } \\
& \underset{j \rightarrow \infty}{\limsup } \mu\left(\zeta_{j}\right)\left|\psi^{\prime}\left(\zeta_{j}\right)\right|\left(\int_{D_{\varphi\left(\zeta_{j}\right)}(r)} \sigma d A\right)^{-1 / p} .
\end{aligned}
$$

Combining (15) and (19), we have that

$$
\begin{aligned}
\left\|W_{\psi, \varphi}\right\|_{e, \mathscr{A}^{p}(\sigma) \rightarrow \mathscr{B}_{\mu}} \geq & \limsup _{|\varphi(z)| \rightarrow 1} \mu(z)\left|\psi^{\prime}(z)\right|\left(\int_{D_{\varphi(z)}(r)} \sigma d A\right)^{-1 / p} \\
& +\limsup _{|\varphi(z)| \rightarrow 1} \frac{\mu(z)\left|\psi(z) \varphi^{\prime}(z)\right|}{1-|\varphi(z)|^{2}} \\
& \cdot\left(\int_{D_{\varphi(z)}(r)} \sigma d A\right)^{-1 / p} .
\end{aligned}
$$


Upper bound. Let $f \in \mathscr{A}^{p}(\sigma)$ be such that $\|f\|_{\mathscr{A}^{p}(\sigma)} \leq 1$. Let

$$
L_{j} f(z)=f\left(a_{j} z\right),
$$

where $a_{j}=j /(j+1)$.

Then, by Theorem 6.1 in [3], we have that $L_{j}: \mathscr{A}^{p}(\sigma)$ $\longrightarrow \mathscr{A}^{p}(\sigma)$ is compact. Since $W_{\psi, \varphi}: \mathscr{A}^{p}(\sigma) \longrightarrow \mathscr{B}_{\mu}$ is bounded, so $W_{\psi, \varphi} L_{j}: \mathscr{A}^{P}(\sigma) \longrightarrow \mathscr{B}_{\mu}$ is compact. Thus, for fixed $r$ in $(0,1)$, we have that

$$
\begin{aligned}
& \left\|W_{\psi, \varphi}\right\|_{e, \mathscr{A}^{p}(\sigma) \rightarrow \mathscr{B}_{\mu}} \leq \sup _{\|f\|_{\mathscr{A P}(\sigma)} \leq 1}\left\|W_{\psi, \varphi}\left(I-L_{j}\right) f\right\|_{\mathscr{B}_{\mu}} \\
& \leq \sup _{\|f\|_{\| \mathcal{P}(\sigma)} \leq 1}\left[\left|W_{\psi, \varphi}\left(I-L_{j}\right) f(0)\right|+\sup _{z \in \mathbb{D}} \mu(z) \mid\right. \\
& \left.\cdot\left(W_{\psi, \varphi}\left(I-L_{j}\right) f\right)^{\prime}(z) \mid\right] \\
& \leq \sup _{\|f\|_{d P^{p}(\sigma) \leq 1}}\left[\left|W_{\psi, \varphi}\left(I-L_{j}\right) f(0)\right|+\sup _{|\varphi(z)| \leq r} \mu(z) \mid\right. \\
& \cdot\left(W_{\psi, \varphi}\left(I-L_{j}\right) f\right)^{\prime}(z)\left|+\sup _{|\varphi(z)|>r} \mu(z)\right| \\
& \left.\cdot\left(W_{\psi, \varphi}\left(I-L_{j}\right) f\right)^{\prime}(z) \mid\right] \text {, }
\end{aligned}
$$

where $I$ is the identity operator on $\mathscr{A}^{p}(\sigma)$. Now

$$
\begin{aligned}
\left|\left(W_{\psi, \varphi}\left(I-L_{j}\right) f\right)^{\prime}(z) \leq\right| & \left|\psi^{\prime}(z)\right|\left|f(\varphi(z))-f\left(a_{j} \varphi(z)\right)\right|+\mid \psi(z) \varphi^{\prime}(z) \| \\
& \cdot\left\{f^{\prime}(\varphi(z))-a_{j} f^{\prime}\left(a_{j} \varphi(z)\right)\right\} \mid \\
\leq & \left|\psi^{\prime}(z)\right|\left|f(\varphi(z))-f\left(a_{j} \varphi(z)\right)\right| \\
& +\frac{\left|\psi(z) \varphi^{\prime}(z)\right|}{j+1}\left|f^{\prime}\left(a_{j} \varphi(z)\right)\right| \\
& +\left|\psi(z) \varphi^{\prime}(z)\right|\left|f^{\prime}(\varphi(z))-f^{\prime}\left(a_{j} \varphi(z)\right)\right| .
\end{aligned}
$$

Let $|\varphi(z)| \leq r$ and $\zeta=\varphi(z)$. Let $\Gamma=\left[a_{j} \zeta, \zeta\right]$ be the line segment from $a_{j} \zeta$ to $\zeta$. Then, $\Gamma \subset D(0, r)$, where $D(0, r)=$ $\{z:|z| \leq r\}$. Thus, by Lemma 2, we have that

$$
\begin{aligned}
\left|f^{\prime}(\zeta)-f^{\prime}\left(a_{j} \zeta\right)\right| & =\left|\int_{\Gamma} f^{\prime \prime}(\lambda) d \lambda\right| \leq \frac{|\zeta|}{j+1} \sup _{\lambda \in D(0, r)}\left|f^{\prime \prime}(\lambda)\right| \\
& \lesssim \frac{|\zeta|}{j+1} \sup _{\lambda \in D(0, r)} \frac{\left(\int_{D_{\lambda}(r)} \sigma d A\right)^{-1 / p}}{\left(1-|\lambda|^{2}\right)^{2}}\|f\|_{A^{p}(\sigma)} .
\end{aligned}
$$

Again, let $\gamma_{0}=(\eta+2) p_{0} / p-2$. Then, by Lemma 2 , we have that

$$
1=\left|K_{\zeta}^{\gamma_{0}}(0)\right| \leq C\left(\int_{D_{0}(1 / 2)} \sigma d A\right)^{-1 / p}\left\|K_{\zeta}^{\gamma_{0}}\right\|_{A^{p}(\sigma)}=\frac{\left(\int_{D_{\zeta}(r)} \sigma d A\right)^{1 / p}}{\left(1-|\zeta|^{2}\right)^{\gamma_{0}+2}} .
$$

Thus,

$$
\frac{\left(1-|\zeta|^{2}\right)^{(\eta+2) p_{0} / p}}{\left(\int_{D_{\zeta}(r)} \sigma d A\right)^{1 / p}} \leq \frac{\left(1-|\zeta|^{2}\right)^{\gamma_{0}+2}}{\left(\int_{D_{\zeta}(r)} \sigma d A\right)^{1 / p}} \leq 1 .
$$

Thus, from (24) and (26), we have that

$$
\begin{aligned}
& \left|f^{\prime}(\zeta)-f^{\prime}\left(a_{j} \zeta\right)\right| \\
& \quad \leq \frac{|\zeta|}{j+1} \sup _{\lambda \in D(0, r)} \frac{1}{\left(1-|\lambda|^{2}\right)^{4+(\eta+2) p_{0} / p}} \frac{\left(1-|\lambda|^{2}\right)^{2+(\eta+2) p_{0} / p}}{\left(\int_{D_{\lambda}(r)} \sigma d A\right)^{1 / p}}\|f\|_{A^{p}(\sigma)} \\
& \quad \leq \frac{|\zeta|}{j+1} \frac{1}{\left(1-|r|^{2}\right)^{4+(\eta+2) p_{0} / p}\|f\|_{A^{p}(\sigma)} .}
\end{aligned}
$$

Similarly, we can show that

$$
\left|f(\zeta)-f\left(a_{j} \zeta\right)\right| \lesssim \frac{|\zeta|}{j+1} \frac{1}{\left(1-|r|^{2}\right)^{3+(\eta+2) p_{0} / p}}\|f\|_{\mathscr{A}^{p}(\sigma)} .
$$

Also, by Lemma 2 and equation (26), we have that

$$
\begin{aligned}
\sup _{|\varphi(z)| \leq r}\left|f^{\prime}\left(a_{j} \zeta\right)\right| & \lesssim \sup _{|\varphi(z)| \leq r} \frac{\left(\int_{D_{a_{j} \varphi(z)}(r)} \sigma d A\right)^{-1 / p}}{1-a_{j}^{2}|\varphi(z)|^{2}}\|f\|_{\mathscr{A}^{p}(\sigma)} \\
& \lesssim \frac{1}{\left(1-a_{j}^{2}|r|^{2}\right)^{1+(\eta+2) p_{0} / p}}\|f\|_{\mathscr{A}^{p}(\sigma)} .
\end{aligned}
$$

Since $W_{\psi, \varphi}: \mathscr{A}^{p}(\sigma) \longrightarrow \mathscr{B}_{\mu}$ is bounded, so

$$
\left\|W_{\psi, \varphi} f\right\|_{\mathscr{B}_{\mu}} \leqslant\|f\|_{\mathscr{A}^{p}(\sigma)}
$$

for each $f \in \mathscr{A}^{P}(\sigma)$. By taking, respectively, $f(z)=1$ and $f(z)=z$ and using the fact that $|\varphi(z)|<1$, we have that

$$
M_{1}=\sup _{z \in \mathbb{D}} \mu(z)\left|\psi^{\prime}(z)\right|<\infty \text { and } M_{2}=\sup _{z \in \mathbb{D}} \mu(z)\left|\psi(z) \varphi^{\prime}(z)\right|<\infty .
$$

Combining (23) and (27)-(29), we have that

$$
\sup _{\left|f \|_{\alpha p^{(\sigma)}} \leq 1\right| \varphi(z) \mid \leq r} \sup _{\mid \leq r} \mu(z)\left|\left(W_{\psi, \varphi}\left(I-L_{j}\right) f\right)^{\prime}(z)\right| \longrightarrow 0 \text { as } j \longrightarrow \infty \text {. }
$$


Using (28), we have that

$$
\begin{aligned}
\left|W_{\psi, \varphi}\left(I-L_{j}\right) f(0)\right| & =\left|\psi(0) f(\varphi(0))-\psi(0) f\left(a_{j} \varphi(0)\right)\right| \\
& \lesssim \frac{|\psi(0) \varphi(0)|}{j+1} \frac{\|f\|_{\mathscr{A}^{p}(\sigma)}}{\left(1-r^{2}\right)^{3+(\eta+2) p_{0} / p}}
\end{aligned}
$$

Using (31), (32), and (33), we have that

$$
\sup _{\|f\|_{\mathcal{S P}(\sigma)} \leq 1|\varphi(z)| \leq r} \sup _{\mid}\left[\left|W_{\psi, \varphi}\left(I-L_{j}\right) f(0)\right|+\left|\left(W_{\psi, \varphi}\left(I-L_{j}\right) f\right)^{\prime}(z)\right|\right] \longrightarrow 0
$$

as $j \longrightarrow \infty$. The last term in the right-hand side of (22) is dominated by

$$
\begin{aligned}
& \sup _{|\varphi(z)|>r} \mu(z)\left|\psi^{\prime}(z)\right|\left\{|f(\varphi(z))|+\left|f\left(a_{j} \varphi(z)\right)\right|\right\} \\
& \quad+\sup _{|\varphi(z)|>r} \mu(z)\left|\psi(z) \varphi^{\prime}(z)\right|\left\{\left|f^{\prime}(\varphi(z))\right|+a_{j}\left|f^{\prime}\left(a_{j} \varphi(z)\right)\right|\right\},
\end{aligned}
$$

which is further dominated by a constant multiple of

$$
\begin{aligned}
\sup _{|\varphi(z)|>r} & \mu(z)\left|\psi^{\prime}(z)\right| \\
& \cdot\left\{\left(\int_{D_{\varphi(z)}(r)} \sigma d A\right)^{-1 / p}+\left(\int_{D_{a_{j} \varphi(z)}(r)} \sigma d A\right)^{-1 / p}\right\}\|f\|_{\mathscr{A}^{p}(\sigma)} \\
& +\sup _{|\varphi(z)|>r} \mu(z)\left|\psi(z) \varphi^{\prime}(z)\right| \\
& \left\{\frac{\left(\int_{D_{\varphi(z)}(r)} \sigma d A\right)^{-1 / p}}{1-|\varphi(z)|^{2}}+\frac{\left(\int_{D_{a_{j} \varphi(z)}(r)} \sigma d A\right)^{-1 / p}}{1-a_{j}^{2}|\varphi(z)|^{2}}\right\}\|f\|_{\mathscr{A}^{p}(\sigma)} .
\end{aligned}
$$

Letting $j \longrightarrow \infty$ in (36), we get

$$
\begin{aligned}
& \underset{j \rightarrow \infty}{\limsup } \sup _{\|f\|_{\mathscr{A P}(\sigma)} \leq 1|\varphi(z)|>r} \sup _{j(z)\left|\left(W_{\psi, \varphi}\left(I-L_{k}\right) f\right)^{\prime}(z)\right|} \\
& \quad \leq \sup _{|\varphi(z)|>r} \mu(z)\left|\psi^{\prime}(z)\right|\left(\int_{D_{\varphi(z)}(r)} \sigma d A\right)^{-1 / p} \\
& \quad+\sup _{|\varphi(z)|>r} \mu(z)\left|\psi(z) \varphi^{\prime}(z)\right| \frac{\left(\int_{D_{\varphi(z)}(r)} \sigma d A\right)^{-1 / p}}{1-|\varphi(z)|^{2}} .
\end{aligned}
$$

Using (34) and (37) in (22), we have that

$$
\begin{aligned}
\left\|W_{\psi, \varphi}\right\|_{e, \mathscr{A}^{p}(\sigma) \rightarrow \mathscr{B}_{\mu}} \leqslant & \sup _{|\varphi(z)|>r} \mu(z)\left|\psi^{\prime}(z)\right|\left(\int_{D_{\varphi(z)}(r)} \sigma d A\right)^{-1 / p} \\
& +\sup _{|\varphi(z)|>r} \mu(z) \mid \frac{\psi(z) \varphi^{\prime}(z) \mid}{1-|\varphi(z)|^{2}}\left(\int_{D_{\varphi(z)}(r)} \sigma d A\right)^{-1 / p} .
\end{aligned}
$$

Finally, letting $r \longrightarrow 1$, then we get

$$
\begin{aligned}
\left\|W_{\psi, \varphi}\right\|_{e, \mathscr{A}^{p}(\sigma) \rightarrow \mathscr{B}_{\mu}} \leq & \limsup _{|\varphi(z)| \rightarrow 1} \mu(z)\left|\psi^{\prime}(z)\right|\left(\int_{D_{\varphi(z)}(r)} \sigma d A\right)^{-1 / p} \\
& +\limsup _{|\varphi(z)| \rightarrow 1} \mu(z) \frac{\left|\psi(z) \varphi^{\prime}(z)\right|}{1-|\varphi(z)|^{2}}\left(\int_{D_{\varphi(z)}(r)} \sigma d A\right)^{-1 / p} .
\end{aligned}
$$

Combining (20) and (39), we get the desired result.

Corollary 5. Let $p \in(1, \infty), \psi \in H(\mathbb{D})$, and $\varphi$ be a holomorphic self-map of $\mathbb{D}$, such that $\|\varphi\|_{\infty}=1$. Let $W_{\psi, \varphi}: \mathscr{A}^{p}(\sigma) \longrightarrow \mathscr{B}_{\mu}$ is bounded. Then, $W_{\psi, \varphi}: \mathscr{A}^{P}(\sigma) \longrightarrow \mathscr{B}_{\mu}$ is compact if and only if the following conditions are satisfied:

(1) $\limsup _{|\varphi(z)| \rightarrow 1} \mu(z)\left|\psi^{\prime}(z)\right|\left(\int_{D_{\varphi(z)}(r)} \sigma d A\right)^{-1 / p}=0$

(2) $\limsup _{|\varphi(z)| \rightarrow 1} \mu(z) \mid\left(\left.\psi(z) \varphi^{\prime}(z)|/ 1-| \varphi(z)\right|^{2}\right)$

$$
\left(\int_{D_{\varphi(z)}(r)} \sigma d A\right)^{-1 / p}=0
$$

\section{Data Availability}

The data used to support the findings of this study are included within the article.

\section{Conflicts of Interest}

The authors declare that they have no conflicts of interest.

\section{Acknowledgments}

We would like to thank the anonymous referee for pointing several errors in the earlier version of the paper. The second author is thankful to DST(SERB) for the project grant (Grant No. MTR/2018/000479) under MATRICS scheme.

\section{References}

[1] D. Békollé, "Inégalités à poids pour le projecteur de Bergman dans la boule unité de $\mathbb{C}^{n}$," Studia Mathematica, vol. 71, no. 3, pp. 305-323, 1982.

[2] O. Constantin, "Discretizations of integral operators and atomic decomposition in vector-valued weighted Bergman spaces," Integral Equations and Operator Theory, vol. 59, no. 4, pp. 523-554, 2007. 
[3] O. Constantin, "Carleson embeddings and some classes of operators on weighted Bergman spaces," Journal of Mathematical Analysis and Applications, vol. 365, no. 2, pp. 668-682, 2010.

[4] C. C. Cowen and B. D. MacCluer, Composition Operators on Spaces of Analytic Functions, CRC Press, Boca Raton, New York, 1995.

[5] K. Madigan and A. Matheson, "Compact composition operators on the Bloch space," Transactions of the American Mathematical Society, vol. 347, no. 7, pp. 2679-2687, 1995.

[6] A. K. Sharma, "Products of multiplication, composition and differentiation between weighted Bergman-Nevanlinna and Bloch-type spaces," Turkish Journal of Mathematics, vol. 35, pp. 275-291, 2011.

[7] A. K. Sharma and S. Ueki, "Composition operators from Nevanlinna type spaces to Bloch type spaces," Banach Journal of Mathematical Analysis, vol. 6, no. 1, pp. 112-123, 2012.

[8] A. K. Sharma and S. Ueki, "Composition operators between weighted Bergman spaces with admissible Bekolle weights," Banach Journal of Mathematical Analysis, vol. 8, no. 1, pp. 64-88, 2014.

[9] S. Stevic and A. K. Sharma, "Composition operators from the space of Cauchy transforms to Bloch and the little Blochtype spaces on the unit disk," Applied Mathematics and Computation, vol. 217, no. 24, pp. 10187-10194, 2011.

[10] S. Stević, A. K. Sharma, and A. Bhat, "Products of multiplication composition and differentiation operators on weighted Bergman spaces," Applied Mathematics and Computation, vol. 217, no. 20, pp. 8115-8125, 2011.

[11] S. Stević, A. K. Sharma, and A. Bhat, "Essential norm of products of multiplication composition and differentiation operators on weighted Bergman spaces," Applied Mathematics and Computation, vol. 218, no. 6, pp. 2386-2397, 2011.

[12] S. Stević and A. K. Sharma, "Weighted composition operators from weighted Bergman spaces with Békollé weights to Blochtype spaces," Journal of Inequalities and Applications, vol. 2015, no. 1, Article ID 337, 2015. 